\title{
Optimal coupling in noisy feed forward leaky integrate and fire
} network

\author{
László Zalányi*1, Zoltán Somogyvári² and Péter Érdi ${ }^{1,2}$
}

Address: ${ }^{1}$ Department of Biophysics, KFKI Research Institute for Particle and Nuclear Physics of the Hungarian Academy of Sciences, Budapest, Hungary and ${ }^{2}$ Center for Complex Systems Studies, Kalamazoo College, Kalamazoo, MI, USA

Email: László Zalányi* - zala@rmki.kfki.hu

* Corresponding author

from Eighteenth Annual Computational Neuroscience Meeting: CNS*2009

Berlin, Germany. 18-23 July 2009

Published: I 3 July 2009

BMC Neuroscience 2009, I0(Suppl I):P302 doi:I0.I I86/I47|-2202-I0-SI-P302

This abstract is available from: http://www.biomedcentral.com//47/-2202//0/SI/P302

(c) 2009 Zalányi et al; licensee BioMed Central Ltd.

We study the stochastic resonance (SR) phenomenon in feed-forward networks of leaky integrate and fire (LIF) neurons. It is shown for various input frequencies, amplitudes and network sizes that the appropriate coupling strength can improve the output signal to noise ratio (SNR). We demonstrate that the value of the optimal coupling strength in the content of SR depends primarily on the absolute refractory period. Other circumstances, signal frequency, amplitude and network size play minor role to determine this value (see Figure 1), consequently it is possible to optimally pretune the system. The optimal coupling strength jumps to discrete values as the noise increases and we discuss the background of this phenomenon.

\section{Acknowledgements}

This study was supported by the grant EU FP6 Programme IST-4-0278I9IP.

\section{References}

I. Shimokawa T, Rogel A, Pakdaman K, Sato S: Stochastic resonance and spike-timing precision in an ensemble of leaky integrate and fire neuron models. Phys Rev E 1999, 59:3461-3470.

2. Zhangcai L, Youguo Q: Stochastic resonance driven by timemodulated neurotransmitter random point trains. Phys Rev Lett 2003, $91: 208103$.
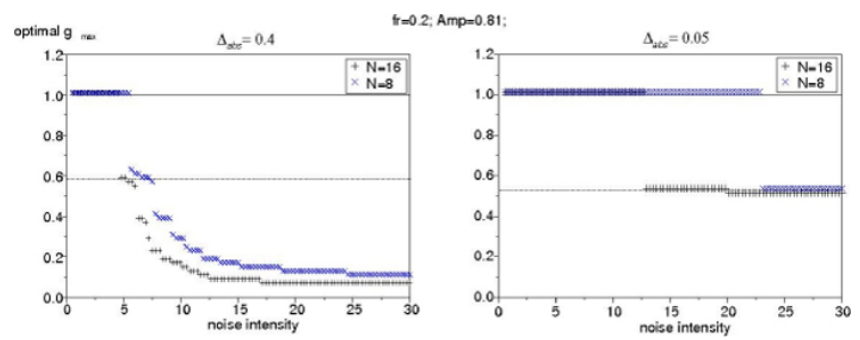

Figure I

Optimal coupling strength as the function of noise intensity with different absolute refractory period. Dotted lines help the comparison of the first optimal coupling values. 